\title{
Cambios en el uso de anticonceptivos, embarazos no planificados e hijos en adolescentes chilenas entre 1997 y 2018
}

\section{Changes in the use of contraceptives, unplanned pregnancies and children in Chilean adolescents between 1997 and 2018}

\section{Ingrid Leal y Temistocles Molina*}

Centro de Medicina Reproductiva y Desarrollo Integral del Adolescente, Facultad de Medicina de la Universidad de Chile, Santiago, Chile

\section{Resumen}

Introducción: El embarazo en las adolescentes ha persistido como un problema de salud pública y social en Chile, afectando a las adolescentes más vulnerables y reflejando las desigualdades. Objetivo: Describir los cambios en el uso de anticonceptivos, embarazos no planificados e hijos en adolescentes chilenas entre 1997 y 2018. Método: Estudio ecológico de series temporales entre los años 1997 y 2018. Se midió la asociación entre variables sociodemográficas y efectividad del método anticonceptivo para los años 2006 y 2018, mediante la prueba estadística de Rao-Scott. Se estimaron tendencias del porcentaje de uso de métodos anticonceptivos según su eficacia y presencia de embarazos no planificados e hijos con modelos de regresión lineal (método de Prais-Winsten). Resultados: Se observó un aumento en la frecuencia de adolescentes sexualmente activas y en el uso de anticonceptivos, especialmente píldoras e inyectables. La serie temporal de uso de anticonceptivos fue no estacionaria, la tendencia fue creciente (coeficiente: 4,59; intervalo de confianza del 95\% [IC95\%]: 3,43-5,74; $p=0,001$ ), aumentando 4,59\% cada 3 años. Las series de embarazos no planificados y tener hijos fueron series temporales no estacionarias y ambas presentaron una tendencia decreciente (coeficiente: $-4,78$; IC95\%: -6,32 a $-3,24$; $p=0,002$; y coeficiente: $-3,93$; IC95\%: $-6,18$ a -1,68; $p=0,008)$, disminuyendo un 4,78\% y un 3,93\%, respectivamente, cada 3 años. Conclusiones: El aumento en el uso de anticonceptivos en adolescentes ha ido unido a una importante disminución en los embarazos no planificados y los hijos en esta población.

Palabras clave: Embarazo adolescente. Anticoncepción. Conducta sexual.

\section{Abstract}

Introduction: Adolescent pregnancy has persisted as a public and social health problem in Chile, affecting the most vulnerable adolescents and reflecting inequalities. Objective: To describe the changes in the use of contraceptives, unplanned pregnancies and children in Chilean adolescents between 1997 and 2018. Method: Ecological study of time series between the years 1997 and 2018. The association between sociodemographic variables and the effectiveness of the contraceptive method for the years 2006 and 2018 was measured using the Rao-Scott statistical test. Trends in the percentage of use of contraceptive methods were estimated according to their efficacy and presence of unplanned pregnancies and children with linear regression models (Prais-Winsten method). Results: An increase in the frequency of sexually active adolescents and the use of contraceptives, especially pills and injectables, was observed. The time series of contraceptive use was non-stationary, the trend was increasing (coefficient: 4.59 ; 95\% confidence interval [95\% Cl]: 3.43-5.74; $p=0.001$ ), increasing $4.59 \%$

Disponible en internet: 27-10-2021 Rev Chil Obstet Ginecol. 2021;86(4):360-367

www. rechog.com CC BY-NC-ND (https://creativecommons.org/licenses/by-nc-nd/4.0/). 
every three years. The time series, unplanned pregnancies and having children were non-stationary time series, both showed a decreasing trend (coefficient: -4.78 ; $95 \% \mathrm{Cl}$ : -6.32 to $-3.24 ; p=0.002$; and coefficient: $-3.93 ; 95 \% \mathrm{Cl}$ : -6.18 to -1.68 ; $p=0.008$ ), decreasing $4.78 \%$ and $3.93 \%$, respectively, every 3 years. Conclusions: The increase in the use of contraceptives in adolescents has been linked to a significant decrease in unplanned pregnancies and children in this population.

Key words: Adolescent pregnancy. Contraception. Sexual behavior.

\section{Introducción}

El embarazo en las adolescentes ha persistido como un problema de salud pública y social en Latinoamérica, con diferencias entre y dentro de los países, afectando a las adolescentes más vulnerables y reflejando las desigualdades ${ }^{1,2}$. En Chile, la tasa específica de fecundidad adolescente (TFA) ha disminuido durante la última década hasta llegar en el año 2017 a 26,5 por cada 1000 adolescentes entre 15 y 19 años, muy por debajo de los 66,5 y los 46 nacimientos por cada 1000 niñas promedio en los países de Latinoamérica y el Caribe y en todo el mundo, respectivamente ${ }^{3,4}$. Chile es el único país de Sudamérica que se ha mantenido con una baja tasa de fecundidad adolescente, según un estudio que midió la disminución de esta en 32 países de América'.

Algunas estrategias que pueden haber influido en la mejora de los indicadores de fecundidad adolescente son la implementación de los llamados «espacios amigables», servicios de atención en salud diferenciados para adolescentes con énfasis en la salud sexual y reproductiva (SSR) ${ }^{5,6}$, y la Ley 20.418 del año 2010, la cual fija las normas sobre información, orientación y prestaciones en materia de regulación de la fertilidad, y establece el derecho a la educación sexual, la información y la confidencialidad independientemente de la edad ${ }^{7}$. Ambas estrategias, junto con los cambios culturales que se han venido produciendo en las últimas décadas, podrían haber influido en las decisiones reproductivas que toman las adolescentes, como el inicio de la actividad sexual, el uso de anticonceptivos, la continuidad o la interrupción de un embarazo, entre otras, que tienen un impacto en la salud, la trayectoria escolar y las perspectivas de empleo, así como en la transición a la adultez ${ }^{8-11}$.

De acuerdo con la literatura, una estrategia altamente efectiva para la disminución de los embarazos adolescentes es el acceso a los anticonceptivos, especialmente a los de acción prolongada (LARC, long action reversible contraceptive), como los dispositivos intrauterinos (DIU) y los implantes, que han demostrado ser muy efectivos (menos de 0,8 fallas por cada 100 mujeres en 1 año) y altamente recomendables para adolescentes, contribuyendo a disminuir los embarazos no deseados, los abortos inseguros y las complicaciones relacionadas con el aborto, y las muertes maternas ${ }^{12-14}$. Los estudios muestran cómo ha ido aumentando la tendencia al uso de LARC en mujeres jóvenes y nulíparas, sobre todo en los países desarrollados $^{15-17}$. A pesar de la sólida evidencia que respalda su uso de primera línea, la mayoría de las adolescentes en Latinoamérica y Chile continúan usando métodos menos efectivos ${ }^{18,19}$.

Se han descrito múltiples barreras para el acceso de las adolescentes a los LARC $^{20,21}$. Algunas revisiones sistemáticas han mostrado las diferencias y las inequidades de acceso a los LARC en Latinoamérica ${ }^{14,19}$. La literatura indica que las adolescentes tienden a elegir más implantes que DIU, a diferencia de las mujeres mayores ${ }^{22}$. En Chile, desde mediados de la década de 2000 se han introducido fuertemente los implantes anticonceptivos en el sistema público de salud y están disponibles los DIU con cobre, y en los últimos años se han incorporado los DIU medicados. A pesar de esta disponibilidad, los datos del Departamento de Estadísticas e Información de Salud señalan que el $67 \%$ de las adolescentes que iniciaron un método anticonceptivo en el sistema público de salud en el año 2018 lo hicieron con un método efectivo de corta duración, como píldoras o inyectables ${ }^{12,19}$. Un estudio que abarcó varios países halló un aumento en el uso de anticonceptivos por las adolescentes en comparación con las adultas, pero este uso era por periodos más cortos y reportaban más fallas ${ }^{23}$.

Algunos datos sobre la SSR para personas entre 15 y 29 años han sido recogidos por las Encuestas Nacionales de la Juventud, realizadas por el Instituto Nacional de la Juventud (INJUV). Según la novena encuesta, del año 2018, de las adolescentes entre 15 y 19 años sexualmente activas, el $86 \%$ reporta el uso de algún anticonceptivo en la última relación sexual. En la última década, estas encuestas han mostrado un aumento del uso de anticonceptivos, pasando de un $58,3 \%$ en el año 2009 a un $84,5 \%$ en el año 2018 en la población de 15 a 29 años ${ }^{24,25}$. En Chile, a diferencia de otros países latinoamericanos, no existen encuestas 
de fecundidad que orienten a conocer las necesidades insatisfechas en anticoncepción o las decisiones reproductivas que toma la población adolescente y los resultados de estas en la SSR.

El objetivo es describir los cambios en el uso de anticonceptivos, hijos y embarazos no planificados en adolescentes de 15 a 19 años encuestadas en Chile entre 1997 y 2018.

\section{Método}

Estudio ecológico de series temporales. EI INJUV realiza periódicamente la Encuesta Nacional de la Juventud a jóvenes entre 15 y 29 años de edad. La muestra para este estudio fueron las adolescentes de 15 a 19 años de las bases de datos desde la segunda (1997) hasta la novena (2018) encuesta. El periodo de recolección fue entre septiembre y diciembre de cada año. El cálculo del tamaño de muestra con significación nacional, el tipo de muestreo, la selección de las participantes, la recolección de la información y el diseño muestral están descritos en la segunda y hasta la novena Encuesta Nacional de la Juventud. Se consideraron los factores de ponderación de cada encuesta. La muestra seleccionada fue de 218 (1997), 268 (2000), 622 (2003), 573 (2006), 816 (2009), 809 (2012), $829(2015)$ y 528 (2018) adolescentes.

\section{Variables de estudio}

- Variables sociodemográficas: edad en años cumplidos, escolaridad (básica, media, superior), nivel socioeconómico (D/E, C2/C3, $\mathrm{ABC} 1)$, área geográfica (urbana/rural) y estado civil (soltera, casada, conviviente).

- Variables de SSR: sin uso de anticonceptivo (sí/no); uso de lavado vaginal (sí/no), métodos naturales (síl no), coito interrumpido (sí/no), condón femenino (síl no), condón masculino (sí/no), diafragma (sí/no), píldora de emergencia (sí/no), anillo vaginal (sí/no), parche (sí/no), píldora (sí/no), inyectable (sí/no), DIU (síl no), implante (sí/no); hijos (sí/no); embarazo no planificado (sí/no). La variable «efectividad y duración del anticonceptivo" fue construida a partir de la pregunta "¿Qué método anticonceptivo usaste tú o tu pareja en tu última relación sexual?", y en el caso de que se usara más de un método anticonceptivo se consideró el más efectivo. Luego esta variable se clasificó en ${ }^{12}$ :
- Menos efectivos: lavado vaginal, método natural, coito interrumpido, condón femenino, diafragma y condón masculino.

- Efectivos de corta duración: píldora de emergencia, anillo, parche, píldora e inyectable.

- Muy efectivos de larga duración: DIU, implante.

\section{Análisis estadístico}

Se trabajó con análisis estadístico para muestras ponderadas (survey). Se realizó análisis descriptivo para caracterizar la muestra. Se midió la asociación entre variables sociodemográficas y efectividad del anticonceptivo para los años 2006 y 2018, mediante la prueba estadística de Rao-Scott.

Se estimaron tendencias del porcentaje de uso de anticonceptivos según su eficacia, presencia de hijos y embarazos no planificados con modelos de regresión lineal (método de Prais-Winsten).

Los datos del estudio se analizaron con el software estadístico STATA v 12 (StataCorp LP, Texas, USA).

Este estudio no fue sometido a un comité de ética, debido a que los datos provienen de una base de datos de acceso público.

\section{Resultados}

Las características sociodemográficas y reproductivas según el uso y el tipo de anticonceptivo para los años 2006 y 2018 se presentan en la tabla 1. En el año 2006 se observó un mayor porcentaje de hijos (96,64\%), embarazos no planificados $(68,54 \%)$, nivel socioeconómico bajo $(76,17 \%)$ y estado civil casada $(12,18 \%)$ en el grupo de adolescentes usuarias de anticonceptivos «muy efectivos de larga duración», con una diferencia estadísticamente significativa ( $p<0,007$ ). En el año 2018 hubo un mayor porcentaje de hijos (38,78\%), embarazos no planificados (28,57\%), nivel socioeconómico bajo $(65,08 \%)$ y estado civil conviviente $(20,36 \%)$ en el grupo de usuarias de anticonceptivos «muy efectivos", con una diferencia estadísticamente significativa $(p<0,037)$.

La mayor proporción de adolescentes sexualmente activas se encontró en el año 2012, con un 48,94\%, y la menor en el año 1997, con un 31,18\%.

Se observó un aumento progresivo en el uso de anticonceptivos, especialmente desde el año 2012 (88,86\%), con un aumento de cerca del $20 \%$ con respecto a la encuesta anterior del año 2009 (69,41\%), llegando a un $86,92 \%$ de uso de anticonceptivos en 2018. La mayor 
Tabla 1. Características demográficas y reproductivas según el uso de métodos anticonceptivos en adolescentes, años 2006 y 2018

\begin{tabular}{|c|c|c|c|c|c|c|c|c|}
\hline \multirow[t]{3}{*}{ Características } & \multicolumn{4}{|c|}{ Año 2006} & \multicolumn{4}{|c|}{ Año 2018} \\
\hline & \multirow{2}{*}{$\begin{array}{c}\text { Sin uso de } \\
\text { MAC }\end{array}$} & \multicolumn{3}{|c|}{ Uso de MAC } & \multirow{2}{*}{$\begin{array}{l}\text { Sin uso de } \\
\text { MAC }\end{array}$} & \multicolumn{3}{|c|}{ Uso de MAC } \\
\hline & & $\begin{array}{l}\text { Menos } \\
\text { efectivos }\end{array}$ & $\begin{array}{l}\text { Efectivos } \\
\text { de corta } \\
\text { duración }\end{array}$ & $\begin{array}{c}\text { Muy } \\
\text { efectivos de } \\
\text { larga } \\
\text { duración }\end{array}$ & & $\begin{array}{l}\text { Menos } \\
\text { efectivos }\end{array}$ & $\begin{array}{l}\text { Efectivos } \\
\text { de corta } \\
\text { duración }\end{array}$ & $\begin{array}{l}\text { Muy } \\
\text { efectivos } \\
\text { de larga } \\
\text { duración }\end{array}$ \\
\hline $\begin{array}{l}\text { Edad promedio } \\
\text { (IC95\%) }\end{array}$ & $\begin{array}{c}17,24 \\
(17,02-17,45)\end{array}$ & $\begin{array}{c}17,45 \\
(17,22-17,68)\end{array}$ & $\begin{array}{c}18,04 \\
(17,82-18,26)\end{array}$ & $\begin{array}{c}17,99 \\
(17,59-18,40)\end{array}$ & $\begin{array}{c}18,07 \\
(17,70-18,43)\end{array}$ & $\begin{array}{c}17,62 \\
(17,38-17,86)\end{array}$ & $\begin{array}{c}18,20 \\
(18,01-18,40)\end{array}$ & $\begin{array}{c}17,60 \\
(17,14-18,06)\end{array}$ \\
\hline $\mathrm{p}$ & \multicolumn{4}{|c|}{0,001} & \multicolumn{4}{|c|}{0,269} \\
\hline $\begin{array}{c}\text { Hijos } \\
\text { Sí } \\
\text { No }\end{array}$ & $\begin{array}{l}22,2 \\
77,8\end{array}$ & $\begin{array}{c}8,58 \\
91,42\end{array}$ & $\begin{array}{l}22,62 \\
77,38\end{array}$ & $\begin{array}{c}96,64 \\
3,36\end{array}$ & $\begin{array}{l}12,04 \\
87,96\end{array}$ & $\begin{array}{c}2,53 \\
97,47\end{array}$ & $\begin{array}{c}3,42 \\
96,58\end{array}$ & $\begin{array}{l}38,78 \\
61,22\end{array}$ \\
\hline $\mathrm{p}$ & \multicolumn{4}{|c|}{0,001} & \multicolumn{4}{|c|}{0,001} \\
\hline $\begin{array}{l}\text { Embarazos no } \\
\text { planificados } \\
\text { Sí } \\
\text { No }\end{array}$ & $\begin{array}{l}41,79 \\
58,21\end{array}$ & $\begin{array}{c}8,64 \\
91,36\end{array}$ & $\begin{array}{l}20,25 \\
79,75\end{array}$ & $\begin{array}{l}68,54 \\
31,46\end{array}$ & $\begin{array}{l}22,07 \\
77,93\end{array}$ & $\begin{array}{c}6,42 \\
93,58\end{array}$ & $\begin{array}{c}4,03 \\
95,97\end{array}$ & $\begin{array}{l}28,57 \\
71,43\end{array}$ \\
\hline $\mathrm{p}$ & \multicolumn{4}{|c|}{0,001} & \multicolumn{4}{|c|}{0,001} \\
\hline $\begin{array}{l}\text { Escolaridad } \\
\text { Básica } \\
\text { Media } \\
\text { Superior }\end{array}$ & $\begin{array}{c}13,92 \\
80,88 \\
5,20\end{array}$ & $\begin{array}{c}6,72 \\
78,93 \\
14,35\end{array}$ & $\begin{array}{c}4,20 \\
74,51 \\
21,29\end{array}$ & $\begin{array}{c}23,16 \\
70,42 \\
6,42\end{array}$ & $\begin{array}{c}4,02 \\
85,81 \\
10,18\end{array}$ & $\begin{array}{c}3,07 \\
76,85 \\
20,08\end{array}$ & $\begin{array}{c}4,01 \\
76,82 \\
19,17\end{array}$ & $\begin{array}{c}21,85 \\
73,56 \\
4,59\end{array}$ \\
\hline $\mathrm{p}$ & \multicolumn{4}{|c|}{0,001} & \multicolumn{4}{|c|}{0,018} \\
\hline $\begin{array}{l}\text { Nivel } \\
\text { socioeconómico } \\
\text { Bajo } \\
\text { Medio } \\
\text { Alto }\end{array}$ & $\begin{array}{c}54,12 \\
44,08 \\
1,80\end{array}$ & $\begin{array}{c}43,16 \\
48,19 \\
8,64\end{array}$ & $\begin{array}{l}42,41 \\
45,88 \\
11,71\end{array}$ & $\begin{array}{c}76,17 \\
23,83 \\
0\end{array}$ & $\begin{array}{c}39,08 \\
60,92 \\
0\end{array}$ & $\begin{array}{c}34,58 \\
58,83 \\
6,58\end{array}$ & $\begin{array}{c}49,55 \\
48,23 \\
2,22\end{array}$ & $\begin{array}{c}65,08 \\
34,92 \\
0\end{array}$ \\
\hline $\mathrm{p}$ & \multicolumn{4}{|c|}{0,007} & \multicolumn{4}{|c|}{0,037} \\
\hline $\begin{array}{l}\text { Área } \\
\text { geográfica } \\
\text { Rural } \\
\text { Urbana }\end{array}$ & $\begin{array}{l}13,94 \\
86,06\end{array}$ & $\begin{array}{l}14,42 \\
85,58\end{array}$ & $\begin{array}{c}8,41 \\
91,59\end{array}$ & $\begin{array}{l}13,65 \\
86,35\end{array}$ & $\begin{array}{c}8,63 \\
91,37\end{array}$ & $\begin{array}{c}5,74 \\
94,26\end{array}$ & $\begin{array}{l}17,65 \\
82,35\end{array}$ & $\begin{array}{c}7,08 \\
92,92\end{array}$ \\
\hline $\mathrm{p}$ & \multicolumn{4}{|c|}{0,454} & \multicolumn{4}{|c|}{0,014} \\
\hline $\begin{array}{l}\text { Estado civil } \\
\text { Soltera } \\
\text { Casada } \\
\text { Conviviente }\end{array}$ & $\begin{array}{c}98,93 \\
1,07\end{array}$ & $\begin{array}{c}99,28 \\
0,72\end{array}$ & $\begin{array}{c}98,24 \\
1,76\end{array}$ & $\begin{array}{l}87,82 \\
12,18\end{array}$ & $\begin{array}{r}93,99 \\
6,01\end{array}$ & $\begin{array}{r}99,04 \\
0,96\end{array}$ & $\begin{array}{r}93,27 \\
6,73\end{array}$ & $\begin{array}{l}79,64 \\
20,36\end{array}$ \\
\hline $\mathrm{p}$ & \multicolumn{4}{|c|}{0,001} & \multicolumn{4}{|c|}{0,001} \\
\hline
\end{tabular}

IC95\%: intervalo de confianza del 95\%; MAC: método anticonceptivo.

proporción de adolescentes sexualmente activas sin uso de anticonceptivos se halló en el año 1997 (44,32\%).

En todas las encuestas se preguntó por el uso de DIU, píldora y condón, mientras que el uso de implante, píldora de emergencia y condón femenino se consultó en las últimas tres encuestas. El uso de diafragma y de lavado vaginal se dejó de preguntar en el año 2003 (Tabla 2).

Los anticonceptivos más usados entre las adolescentes fueron las píldoras (25,67\% en 1997 y $27,43 \%$ en 2018 ), el condón (17,52\% en 1997 y $29,04 \%$ en 2018) y los inyectables $(0,40 \%$ en 2006 y $20,60 \%$ en 


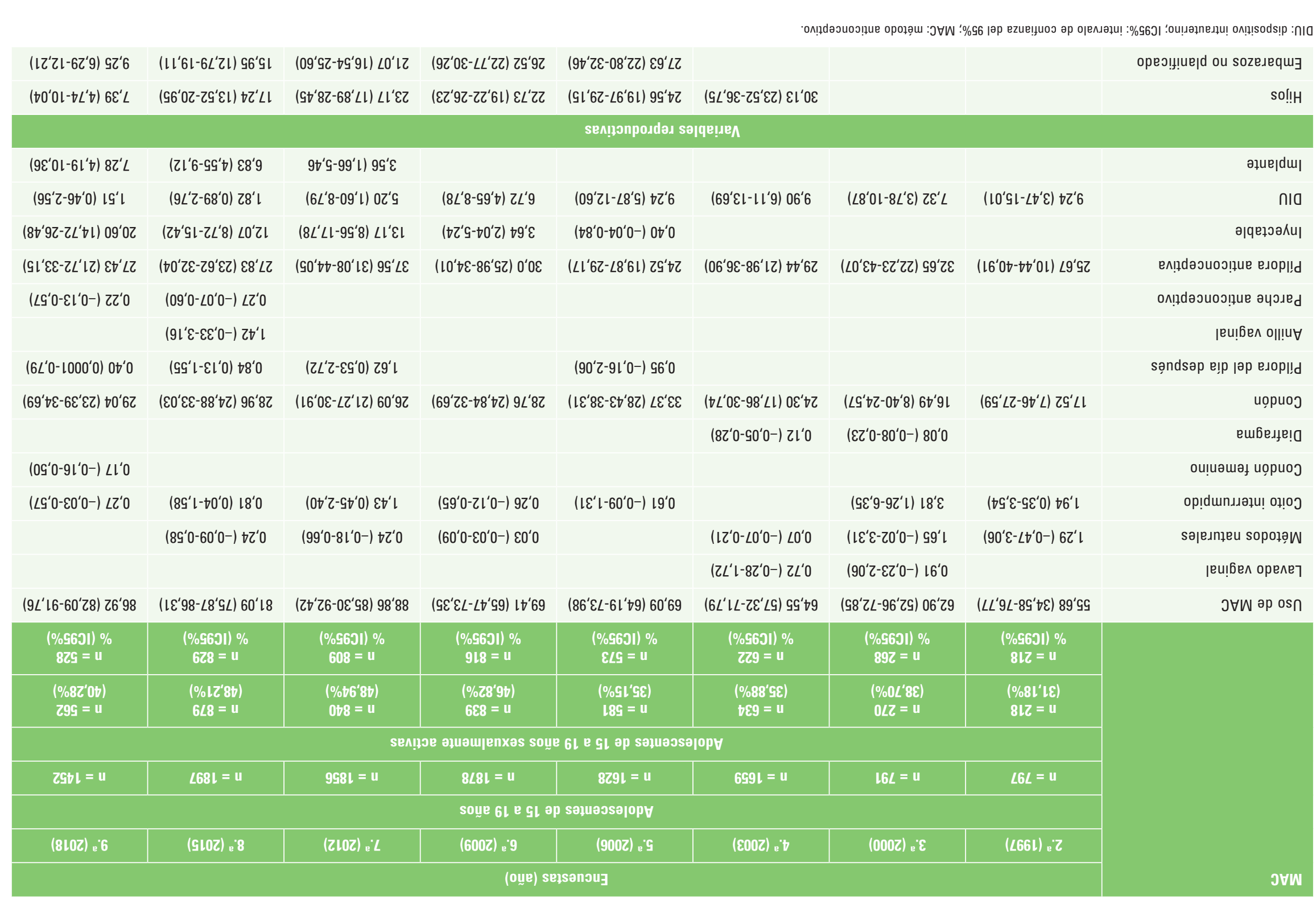

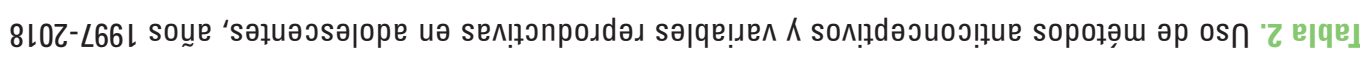


Tabla 3. Uso de métodos anticonceptivos según efectividad en las adolescentes, años 1997-2018

\begin{tabular}{|c|c|c|c|c|c|c|c|c|c|c|c|}
\hline \multirow[t]{3}{*}{ MAC } & \multicolumn{8}{|c|}{ Encuestas (año) } & \multirow{2}{*}{\multicolumn{3}{|c|}{ Regresión de Prais-Winsten }} \\
\hline & $\begin{array}{l}2^{\mathrm{a}} \\
(1997)\end{array}$ & $\begin{array}{l}3 .^{a} \\
(2000)\end{array}$ & $\begin{array}{c}4^{4^{a}} \\
(2003)\end{array}$ & $\begin{array}{l}5{ }^{\mathrm{a}} \\
(2006)\end{array}$ & $\begin{array}{l}6^{\mathrm{a}} \\
(2009)\end{array}$ & $\begin{array}{c}7^{\mathrm{a}} \\
(2012)\end{array}$ & $\begin{array}{l}8^{8}{ }^{a} \\
(2015)\end{array}$ & $\begin{array}{c}9 .^{a} \\
(2018)\end{array}$ & & & \\
\hline & $\begin{array}{c}n= \\
218 \%\end{array}$ & $\begin{array}{c}n= \\
268 \%\end{array}$ & $\begin{array}{c}n= \\
622 \%\end{array}$ & $\begin{array}{c}n= \\
573 \%\end{array}$ & $\begin{array}{c}n= \\
816 \%\end{array}$ & $\begin{array}{c}n= \\
809 \%\end{array}$ & $\begin{array}{c}n= \\
829 \%\end{array}$ & $\begin{array}{c}n= \\
528 \%\end{array}$ & Coef. & p & (IC95\%) \\
\hline $\begin{array}{l}\text { Menos } \\
\text { efectivos }\end{array}$ & 20,76 & 22,93 & 25,21 & 33,98 & 29,06 & 27,75 & 30,01 & 29,48 & 1,29 & 0,001 & $(0,93-1,64)$ \\
\hline $\begin{array}{l}\text { Efectivos } \\
\text { de corta } \\
\text { duración }\end{array}$ & 25,67 & 32,65 & 29,44 & 25,87 & 33,63 & 52,35 & 42,43 & 48,66 & 3,42 & 0,011 & $(1,13-5,70)$ \\
\hline $\begin{array}{l}\text { Muy } \\
\text { efectivos } \\
\text { de larga } \\
\text { duración }\end{array}$ & 9,24 & 7,32 & 9,9 & 9,24 & 6,72 & 8,76 & 8,66 & 8,79 & $-0,02$ & 0,884 & $(-0,33-0,29)$ \\
\hline
\end{tabular}

IC95\%: intervalo de confianza del 95\%; MAC: método anticonceptivo.

2018); estos últimos presentaron un aumento de más del $20 \%$. Se observó una disminución en el uso de DIU, del $9,24 \%$ en 1997 al $1,51 \%$ en 2018 , y un aumento del uso de implante, del 3,56\% en 2012 al 7,28\% en 2018 .

La tabla 3 muestra la serie temporal para el uso de anticonceptivos «menos efectivos», que en el año 2006 presentó un valor atípico (outlier); imputando este valor, la serie presentó una tendencia creciente (coeficiente: 1,29; intervalo de confianza del 95\% [IC95\%]: 0,93$1,64 ; p=0,001)$ y aumentó un $1,29 \%$ cada 3 años. La serie temporal de uso de anticonceptivos «efectivos de corta duración» fue no estacionaria, con una tendencia creciente (coeficiente: 3,42; IC95\%: 1,13-5,70; $p=0,011$ ) y aumentó un $3,42 \%$ cada 3 años.

La figura 1 muestra que la serie temporal de uso de anticonceptivos fue no estacionaria, la tendencia fue creciente (coeficiente: 4,59; IC95\%: 3,43-5,74; $p=0,001$ ) y aumentó un $4,59 \%$ cada 3 años. Respecto a las variables reproductivas, embarazos no planificados y tener hijos, fueron series temporales no estacionarias y presentaron una tendencia decreciente (coeficiente: $-4,78$; IC95\%: $-6,32$ a -3,24; $p=0,002$; y coeficiente: $-3,93$; IC95\%: $-6,18$ a -1,68; $p=0,008$ ), disminuyendo un $4,78 \%$ y un $3,93 \%$, respectivamente, cada 3 años.

\section{Discusión}

Se observó un aumento en el uso de anticonceptivos, en especial de los efectivos de corta duración, como inyectables y píldoras, lo que concuerda con otros estudios que muestran que estos anticonceptivos son los más usados por las adolescentes ${ }^{18,23}$. El

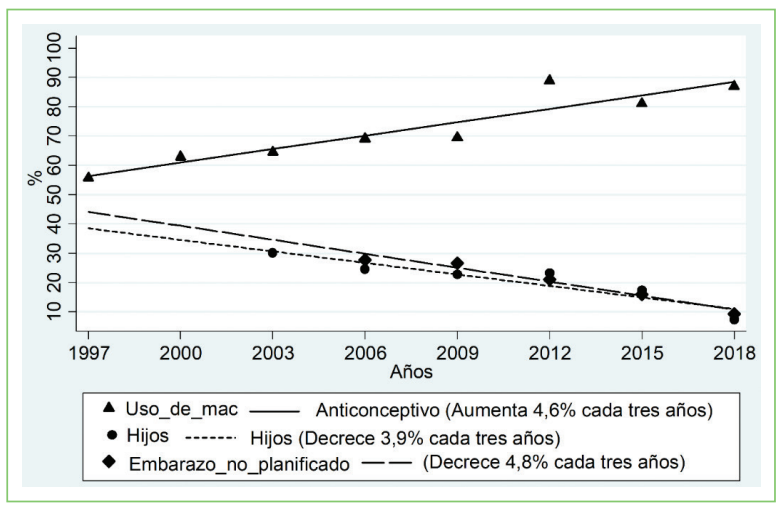

Figura 1. Porcentaje de cambio en el uso de anticonceptivos, embarazos no planificados e hijos en adolescentes chilenas, 1997-2018. MAC: método anticonceptivo.

principal aumento se registró en el año 2012, y si bien en este estudio no se buscó medir la asociación entre las políticas públicas implementadas y la disminución del embarazo adolescente, llama la atención que esta disminución ocurre posterior a la promulgación de la Ley 20.418 y al aumento de los espacios amigables, política pública impulsada a partir del año 2014 que, al hacer un especial énfasis en la SSR, ha aumentado el acceso a los métodos anticonceptivos ${ }^{6,7}$.

Es destacable el aumento del uso de anticonceptivos efectivos sobre los menos efectivos, dado que la efectividad es el principal atributo que buscan las mujeres en un anticonceptivo ${ }^{21}$. Esto denota la creciente preocupación de las adolescentes por evitar el embarazo, 
eligiendo anticonceptivos efectivos que están a su alcance, pero considerando que persisten barreras para acceder a los LARC ${ }^{26}$.

El uso de anticonceptivos muy efectivos de larga duración no mostró variaciones significativas en su conjunto, estando muy por debajo de los de corta duración efectivos o menos efectivos. Los datos por separado mostraron una importante disminución del uso de DIU y un aumento del uso de implantes, los cuales duplicaron su uso en 6 años, lo que refuerza lo descrito en la literatura sobre la tendencia de las adolescentes a elegir implantes más que DIU ${ }^{21,22}$. Los porcentajes de uso de LARC fueron mayores que los reportados en conjunto en Latinoamérica y similares a los informados en los Estados Unidos de América para adolescentes ${ }^{14,15,18}$. Las causas de este menor uso pueden ser la necesidad de un proveedor de salud capacitado para su inicio, los costos iniciales elevados, el consentimiento de un adulto, y las creencias y los mitos relativos a los LARC, entre ellos la idea de la necesidad de paridad probaba para su uso ${ }^{18,20}$. Esto último coincide con lo descrito en los años 2006 y 2018, y con lo reportado en otros estudios, en los que las adolescentes con antecedentes de embarazos e hijos presentan un mayor uso de estos anticonceptivos ${ }^{20,24}$. Ello se podría explicar porque en estas adolescentes la actividad sexual no es confidencial, están insertas en el sistema de salud y pueden iniciar el uso de estos anticonceptivos en el posparto inmediato ${ }^{18,27}$.

Se observó un alto porcentaje de uso únicamente de condón como anticonceptivo, a pesar de su alta tasa de falla y no dependencia directa de las adolescentes, de modo similar a otro estudio, lo que podría reflejar la apertura por parte de los varones a involucrarse en la prevención del embarazo, situación que históricamente ha estado a cargo de las mujeres, y el mayor acceso a este método ${ }^{14}$.

La disminución y la tendencia a la baja de los embarazos no planificados y de los hijos en las adolescentes chilenas concuerda con las cifras informadas en otro estudio y con el aumento del uso de anticonceptivos por parte de esta población, cada vez más activa sexualmente ${ }^{14}$. Esto reafirma la importante contribución que tiene el acceso a anticonceptivos en la disminución de los embarazos no planificados, lo que ha sido destacado en otros estudios y reforzado por distintas organizaciones de abogacía por los derechos sexuales y reproductivos ${ }^{14}$.

A pesar de que se encontró un cada vez menor número de adolescentes que no usaron ningún anticonceptivo, y estas cifras estuvieron por debajo de lo reportado en países de ingresos bajos y medios, pero por encima de las de países como los Estados Unidos de América, sigue siendo un tema preocupante que refleja las necesidades insatisfechas en anticoncepción de este grupo, y dentro de él afecta a las personas más vulnerables, como adolescentes migrantes internacionales, con alguna discapacidad y LGBTIQ $^{14,28}$.

El ligero mayor número de embarazos no planificados en relación a hijos puede explicarse porque no todos los embarazos terminan en el nacimiento de un hijo, ya sea por una pérdida reproductiva espontánea o por un aborto inducido. Probablemente no se obtuvieron todos los embarazos en adolescentes, ya que no todos ellos se refieren como no planificados.

Las limitaciones de este estudio se relacionan con su diseño, que no permite estimar el tiempo de uso, la tasa de descontinuación ni el cambio de anticonceptivo, y tampoco se contó con la información de cada variable de interés en todas las encuestas. Por otro lado, este es el primer estudio de ámbito nacional que muestra la evolución y el cambio en el uso de anticonceptivos por parte de las adolescentes y su relación con la disminución de los embarazos no planificados y de hijos reportados, haciendo una descripción general del fenómeno.

Se han observado cambios en las variables reproductivas de las adolescentes en los últimos 20 años, coincidentes con las políticas públicas en salud con foco en la reducción de la fecundidad adolescente que Chile ha implementado, y con los cambios socioculturales que el país ha experimentado en torno a la sexualidad.

Es necesario contar con una encuesta nacional de fertilidad que permita conocer datos sobre las decisiones y los resultados respecto a la SSR de esta población y en general, visibilizando las necesidades de grupos específicos de mayor vulnerabilidad, para reforzar y mejorar las estrategias de salud pública, como acceso a servicios de SSR, acceso a LARC y educación sexual integral.

\section{Conclusiones}

Los resultados de este estudio muestran un aumento en el uso de métodos anticonceptivos y una disminución en los embarazos no planificados y en los hijos en las adolescentes entre 15 y 19 años. Los métodos anticonceptivos más usados son las píldoras y los inyectables, y se observa un aumento del uso de implantes, en especial en las adolescentes con antecedente de embarazo o hijos. Es necesario realizar 
estudios que permitan incorporar nuevas estrategias que promuevan una SSR saludable.

\section{Financiamiento}

Este estudio fue financiado por el Centro de Medicina Reproductiva y Desarrollo Integral del Adolescente (CEMERA).

\section{Conflicto de intereses}

Los autores no presentan conflictos de intereses.

\section{Responsabilidades éticas}

Protección de personas y animales. Los autores declaran que para esta investigación no se han realizado experimentos en seres humanos ni en animales.

Confidencialidad de los datos. Los autores declaran que en este artículo no aparecen datos de pacientes.

Derecho a la privacidad y consentimiento informado. Los autores declaran que en este artículo no aparecen datos de pacientes.

\section{Bibliografía}

1. Guerrero Núñez J. Disminución desigual de las tasas de fecundidad en adolescentes de 32 países de la Región de las Américas, 1960-2019. Rev Panam Salud Publica. 2020;44:e71.

2. Lavanderos S, Haase J, Riquelme C, Morales A, Martínez A. Embarazo adolescente en Chile: una mirada a la desigualdad sociodemográfica comunal. Rev Chil Obstet Ginecol. 2019;84:490-508.

3. Instituto Nacional de Estadísticas. Publicación Estadísticas Vitales. Cifras provisionales: 2017. Santiago: INE; 2017.

4. Organización Panamericana de la Salud, Fondo de Población de las Naciones Unidas, Fondo de las Naciones Unidas para la Infancia. Acelerar el progreso hacia la reducción del embarazo en la adolescencia en América Latina y el Caribe. Washington, DC: OPS, UNFPA, UNICEF; 2016. p. 56.

5. Ministerio de Salud, Subsecretaría de Redes Asistenciales. Informe de Seguimiento de Programas Sociales: Espacios Amigables. Santiago: MINSAL; 2016.

6. Ministerio de Salud, Subsecretaría de Salud Pública, División Prevención y Control de Enfermedades. Programa Nacional de Salud Integral de Adolescentes y Jóvenes "Nivel Primario de Atención." Santiago: MINSAL; 2018.

7. Ley 20.418. Fija Normas sobre Información, Orientación y Prestaciones en Materia de Regulación de la Fertilidad. Biblioteca del Congreso Nacional (28-01-2010). [Consultado el 5 de mayo de 2012]. Disponible en: http://www.leychile.cl/N?i=1010482\&f=2010-02-02\&p=
8. Molina Gómez AM, Pena Olivera RA, Díaz Amores CE, Antón Soto M. Condicionantes y consecuencias sociales del embarazo en la adolescencia. Rev Cubana Obstet Ginecol. 2019;45:e218.

9. Dides C, Fernández C. Primer Informe de Salud Sexual, Salud Reproductiva y Derechos Humanos en Chile. Santiago: Corporación Miles; 2016.

10. Flores-Valencia ME, Nava-Chapa G, Arenas-Monreal L. Embarazo en la adolescencia en una región de México: un problema de Salud Pública. Rev Salud Publica. 2017;19:374-8.

11. Faisal-Cury A, Tabb KM, Niciunovas G, Cunningham C, Menezes PR, Huang $\mathrm{H}$. Lower education among low-income Brazilian adolescent females is associated with planned pregnancies. Int $\mathrm{J}$ Womens Health. 2017;9:43-8.

12. Trussell J. Contraceptive failure in the United States. Contraception. 2011;83:397-404.

13. Bahamondes L, Villarroel C, Frías Guzmán N, Oizerovich S, Velázquez-Ramírez N, Monteiro I. The use of long-acting reversible contraceptives in Latin America and the Caribbean: current landscape and recommendations. Hum Reprod Open. 2018;(1):hox030.

14. Lindberg LD, Santelli JS, Desai S. Changing patterns of contraceptive use and the decline in rates of pregnancy and birth among U.S. adolescents, 2007-2014. J Adolesc Health. 2018;63:253-6.

15. Patel PR, Abacan A, Smith PB. Trends of contraceptive choices among young women in inner city Houston. J Pediatr Adolesc Gynecol. 2019;32:487-90.

16. Ihongbe TO, Masho SW. Changes in the use of long-acting reversible contraceptive methods among U.S. nulliparous women: results from the 2006-2010, 2011-2013, and 2013-2015 National Survey of Family Growth. J Womens Health (Larchmt). 2017;27:245-52.

17. Hellström A, Gemzell Danielsson K, Kopp Kallner H. Trends in use and attitudes towards contraception in Sweden: results of a nationwide survey. Eur J Contracept Reprod Heal Care. 2019;24:154-60.

18. Ponce de León RG, Ewerling F, Serruya SJ, Silveira MF, Sanhueza A, Moazzam A, et al. Contraceptive use in Latin America and the Caribbean with a focus on long-acting reversible contraceptives: prevalence and inequalities in 23 countries. Lancet Glob Heal. 2019;7:e227-35.

19. Ministerio de Salud, Departamento de Estadísticas e Información en Salud. Población en control, según método de regulación de fertilidad, por Región y Servicio de Salud, SNSS 2018 (datos preliminares). Santiago: MINSAL, DEIS; 2018.

20. Ba NMP, Norris AH, Berlan ED. Mini-review barriers and facilitators to adolescents use of long-acting reversible contraceptives. J Pediatr Adolesc Gynecol. 2016;30:18-22.

21. Diedrich JT, Klein DA, Peipert JF. Long-acting reversible contraception in adolescents: a systematic review and meta-analysis. Am J Obstet Gynecol. 2017;216:364.e1-12.

22. Mestad R, Secura G, Allsworth JE, Madden T, Zhao Q. Adolescent participants in the Contraceptive CHOICE Project. Contraception. 2011;84:493-8.

23. Blanc AK, Tsui AO, Croft TN, Trevitt JL. Patterns and trends in adolescents' contraceptive use and discontinuation in developing countries and comparisons with adult women. Int Perspect Sex Reprod Health. 2009;35:63-71.

24. Aravena A, Alt C, Argote L, Barretto M, Donoso A, Ferreiro J. Sexta Encuesta Nacional de Juventud. Santiago: Instituto Nacional de la Juventud; 2010.

25. Salfate M, Jaqueih MJ, Nuñez C, Westhoff R, Barretto M, Becker I, et al. Novena Encuesta Nacional de la Juventud. Santiago: Instituto Nacional de la Juventud; 2018.

26. Pritt NM, Norris AH, Berlan ED. Barriers and facilitators to adolescents' use of long-acting reversible contraceptives. J Pediatr Adolesc Gynecol. 2017;30:18-22.

27. Chacko MR, Wiemann CM, Buzi RS, Kozinetz CA, Peskin M, Smith PB. Choice of postpartum contraception: factors predisposing pregnant adolescents to choose less effective methods over long-acting reversible contraception. J Adolesc Health. 2016;58:628-35.

28. Sadinsky S. De mal en peor: la pandemia de COVID-19 amenaza aún más la salud y los derechos sexuales y reproductivos de los adolescentes en muchos países. New York: Guttmacher Institute; 2020. 